\title{
Equivalence of the long-wavelength approximation and the truncated Taylor expansion in relativistic Coulomb excitation
}

\author{
B.F. Bayman \\ School of Physics and Astronomy, University of Minnesota, \\ 116 Church Street S.E., Minneapolis, MN 55410, U.S.A. \\ and \\ F.Zardi \\ Istituto Nazionale di Fisica Nucleare and Dipartimento di Fisica, \\ Via Marzolo, 8 I-35131 Padova,Italy.
}

November 11, 2018

\begin{abstract}
The long-wavelength approximation and the truncated Taylor expansion are frequently used in the theory of relativistic Coulomb excitation to obtain multipole expansions of the interaction. It is shown in this note that these two approximations are exactly equivalent.
\end{abstract}

PACS numbers: 25.70.De, 25.75.-q, 24.30.Cz

\section{Introduction}

Relativistic Coulomb excitation is an important tool for the study of several aspects of nuclear structure that cannot be explored through the nuclear interaction [1-7]. An important quantity in the analysis of relativistic Coulomb excitation is the retarded scalar potential[8], at point $\mathbf{r}$ and time $t$, due to a projectile moving with speed $v$ along a straightline trajectory:

$$
\Phi(\mathbf{r}, t)=\frac{Z_{P} e \gamma}{\sqrt{\left(x-b_{x}\right)^{2}+\left(y-b_{y}\right)^{2}+\gamma^{2}(z-v t)^{2}}}
$$

The charge of the projectile is $Z_{P} e$, the impact parameter of the trajectory is $b$, and the angle between the reaction plane and the $\hat{x}-\hat{z}$ plane is $\phi_{b}\left(b_{x}=b \cos \phi_{b}, b_{y}=b \sin \phi_{b}\right)$. To calculate matrix elements of the electromagnetic interaction between nuclear angular momentum eigenstates to be used in coupled-channel time-dependent differential equations, it would be convenient to have a multipole expansion of eq.(1), but unfortunately no exact multipole expansion is available for $\Phi(\mathbf{r}, t)$. 
Alder and Winther [1] have derived an exact multipole expansion for the Fourier transform, $V(\omega, b)$, of $\Phi(\mathbf{r}, t)$. This representation can be used directly to calculate the Born approximation, and has been used in ref. [9] in a coupled integral equation formulation of the Schroedinger equation. However this approach is difficult to implement when many excited states have to be included in the calculation.

Two approximate multipole expansions in the $t$-representation have been developed [5, 6]. They start from two different exact expansions of $\Phi(\mathbf{r}, t)$, and then truncate these expansions in different ways. The purpose of this report is to show that the two approximate expansions so obtained are identical.

\section{The Long-Wavelength Approximation (LWLA)}

If we invert the $V(\omega, b)$ transform of Alder and Winther, we can write a formally exact multipole expansion

$$
\Phi(\mathbf{r}, t)=\frac{\hbar}{2 \pi} \int_{\infty}^{\infty} e^{-i \omega t}\left[\sum_{\lambda \mu} e^{-i \mu \phi_{b}} C_{\lambda, \mu}(\omega) K_{\mu}\left(\frac{|\omega| b}{v \gamma}\right) j_{\lambda}\left(\frac{|\omega|}{c} r\right) Y_{\mu}^{\lambda}(\hat{r}]\right) d \omega
$$

with

$$
C_{\lambda, \mu}(\omega) \equiv \frac{2 Z_{P} e}{\hbar v} \mathcal{G}_{\lambda, \mu}
$$

where

$$
\begin{aligned}
\mathcal{G}_{\lambda, \mu} & \equiv \frac{i^{\lambda+\mu}}{(2 \gamma)^{\mu}}\left(\frac{\omega}{|\omega|}\right)^{\lambda-\mu}\left(\frac{c}{v}\right)^{\lambda} \sqrt{4 \pi(2 \lambda+1)(\lambda-\mu) !(\lambda+\mu) !} \\
& \times \sum_{n} \frac{1}{(2 \gamma)^{2 n}(n+\mu) ! n !(\lambda-\mu-2 n) !}
\end{aligned}
$$

and

$$
j_{\lambda}\left(\frac{|\omega|}{c} r\right)=\sum_{\ell=\lambda, \lambda+2, \ldots}\left(\frac{|\omega|}{c} r\right)^{\ell} \frac{\left(-\frac{1}{2}\right)^{\frac{\ell-\lambda}{2}}}{\left(\frac{\ell-\lambda}{2}\right) !(\ell+\lambda+1) ! !}
$$

If this is substituted into the Alder-Winther expansion, and the coefficient of the $r^{\ell} Y_{\mu}^{\lambda}(\hat{r})$ term is identified, we can write

$$
\Phi(\mathbf{r}, t)=\sum_{\lambda, \mu, \ell(=\lambda, \lambda+2, \ldots)} A_{\mu}^{\lambda, \ell} r^{\ell} Y_{\mu}^{\lambda}(\hat{r})
$$

with

$$
A_{\mu}^{\lambda, \ell}=\frac{\hbar}{2 \pi} \frac{\left(-\frac{1}{2}\right)^{\frac{\ell-\lambda}{2}} e^{-i \mu \phi_{b}}}{\left(\frac{\ell-\lambda}{2}\right) !(\ell+\lambda+1) ! !} \times \int_{-\infty}^{\infty} e^{-i \omega t}\left(\frac{|\omega|}{c}\right)^{\ell} C_{\lambda, \mu}(\omega) K_{\mu}\left(\frac{|\omega| b}{v \gamma}\right) d \omega
$$

The LWLA consists of keeping only the first term in the expansion of the spherical Bessel function, which is equivalent to the approximation

$$
\Phi(\mathbf{r}, t) \sim \sum_{\lambda, \mu} A_{\mu}^{\lambda, \lambda} r^{\lambda} Y_{\mu}^{\lambda}(\hat{r}) \quad \text { LWLA. }
$$

This is the approximation used, e.g., in ref. [6]. 


\section{The Truncated Taylor Expansion (TTEA)}

Another formally exact expansion of $\Phi(\mathbf{r}, t)$ is a Taylor expansion, which can be written

$$
\Phi(\mathbf{r}, t)=\left.\sum_{\ell=0}^{\infty} \frac{1}{\ell !}\left(\mathbf{r} \cdot \nabla_{\mathbf{s}}\right)^{\ell} \Phi(\mathbf{s}, t)\right|_{\mathbf{s}=0}
$$

To express this as a multipole expansion, we write the dot product in terms of Legendre polynomials and spherical harmonics. For any vectors $\mathbf{a}, \mathbf{b}$

$$
\begin{aligned}
& (\mathbf{a} \cdot \mathbf{b})^{\ell}=a^{\ell} b^{\ell}\left(\cos \left(\omega_{a b}\right)\right)^{\ell}=a^{\ell} b^{\ell} \sum_{\lambda=\ell, \ell-2, \ldots} \frac{(2 \lambda+1) 2^{\lambda} \ell !\left(\frac{\ell+\lambda}{2}\right) !}{(\ell+\lambda+1) !\left(\frac{\ell-\lambda}{2}\right) !} P_{\lambda}\left(\cos \left(\omega_{a b}\right)\right) \\
= & a^{\ell} b^{\ell} \sum_{\lambda=\ell, \ell-2, \ldots} \frac{(2 \lambda+1) 2^{\lambda} \ell !\left(\frac{\ell+\lambda}{2}\right) !}{(\ell+\lambda+1) !\left(\frac{\ell-\lambda}{2}\right) !}\left(a^{2}\right)^{\frac{\ell-\lambda}{2}}\left(b^{2}\right)^{\frac{\ell-\lambda}{2}} \times \frac{4 \pi}{2 \lambda+1} \sum_{\mu=-\lambda}^{\lambda} \mathcal{Y}_{\mu}^{\lambda}(\mathbf{a}) \mathcal{Y}_{\mu}^{\lambda *}(\mathbf{b}),
\end{aligned}
$$

with $\mathcal{Y}_{\mu}^{\lambda}(\mathbf{a}) \equiv a^{\lambda} Y_{\mu}^{\lambda}(\hat{a})$, a homogeneous polynomial of degree $\lambda$ in $a_{x}, a_{y}, a_{z}$. If we identify $\mathbf{a}$ with $\mathbf{r}$ and $\mathbf{b}$ with $\nabla_{\mathbf{s}}$, we can write

$$
\left(\mathbf{r} \cdot \nabla_{\mathbf{s}}\right)^{\ell}=\sum_{\lambda=\ell, \ell-2, \ldots} \frac{(2 \lambda+1) 2^{\lambda} \ell !\left(\frac{\ell+\lambda}{2}\right) !}{(\ell+\lambda+1) !\left(\frac{\ell-\lambda}{2}\right) !}\left(r^{2}\right)^{\frac{\ell-\lambda}{2}} \times \frac{4 \pi}{2 \lambda+1} \sum_{\mu=-\lambda}^{\lambda} \mathcal{Y}_{\mu}^{\lambda}(\mathbf{r}) \mathcal{Y}_{\mu}^{\lambda *}\left(\nabla_{\mathbf{s}}\right)\left(\nabla_{s}^{2}\right)^{\frac{\ell-\lambda}{2}},
$$

so that

$$
\Phi(\mathbf{r}, t)=4 \pi \sum_{\lambda=0}^{\infty} \sum_{\ell=\lambda, \lambda+2, \ldots} \frac{2^{\lambda}\left(\frac{\ell+\lambda}{2}\right) !}{(\ell+\lambda+1) !\left(\frac{\ell-\lambda}{2}\right) !}\left(r^{2}\right)^{\frac{\ell-\lambda}{2}} \times\left.\sum_{\mu=-\lambda}^{\lambda} \mathcal{Y}_{\mu}^{\lambda}(\mathbf{r}) \mathcal{Y}_{\mu}^{\lambda *}\left(\nabla_{\mathbf{s}}\right)\left(\nabla_{s}^{2}\right)^{\frac{\ell-\lambda}{2}} \Phi(\mathbf{s}, t)\right|_{\mathbf{s}=0} .
$$

Now if we extract the coefficient of $r^{\ell} Y_{\mu}^{\lambda}(\hat{r})$, we can write

$$
\Phi(\mathbf{r}, t)=\sum_{\lambda, \mu, \ell(=\lambda, \lambda+2, \ldots)} B_{\mu}^{\lambda, \ell} r^{\ell} Y_{\mu}^{\lambda}(\hat{r})
$$

with

$$
B_{\mu}^{\lambda, \ell}=\left.4 \pi \frac{2^{\lambda}\left(\frac{\ell+\lambda}{2}\right) !}{(\ell+\lambda+1) !\left(\frac{\ell-\lambda}{2}\right) !} \mathcal{Y}_{\mu}^{\lambda *}\left(\nabla_{\mathbf{s}}\right)\left(\nabla_{s}^{2}\right)^{\frac{\ell-\lambda}{2}} \Phi(\mathbf{s}, t)\right|_{\mathbf{s}=0}
$$

where $\ell=\lambda, \lambda+2, \ldots$

Since both Equations (4) and (6) hold identically in $\mathbf{r}$, it must be that

$$
A_{\mu}^{\lambda, \ell}=B_{\mu}^{\lambda, \ell}
$$

which implies that

$$
\begin{aligned}
& \int_{-\infty}^{\infty} e^{-i \omega t}\left(\frac{|\omega|}{c}\right)^{\ell} C_{\lambda, \mu}(\omega) K_{\mu}\left(\frac{|\omega| b}{v \gamma}\right) d \omega=\left.e^{i \mu \phi_{b}}\left(-\frac{1}{2}\right)^{\frac{\ell-\lambda}{2}} \frac{8 \pi^{2}}{\hbar}\left[\mathcal{Y}_{\mu}^{\lambda *}\left(\nabla_{\mathbf{s}}\right)\left(\nabla_{s}^{2}\right)^{\frac{\ell-\lambda}{2}}\right] \Phi(\mathbf{s}, t)\right|_{\mathbf{s}=0} \\
& =\left.e^{i \mu \phi_{b}}\left(-\frac{1}{2}\right)^{\frac{\ell-\lambda}{2}} \frac{8 \pi^{2}}{\hbar}\left[\mathcal{Y}_{\mu}^{\lambda *}\left(\nabla_{\mathbf{s}}\right)\left(\nabla_{s}^{2}\right)^{\frac{\ell-\lambda}{2}}\right] \frac{Z_{\mathrm{P}} e \gamma}{\sqrt{\left(s_{x}-b_{x}\right)^{2}+\left(s_{y}-b_{y}\right)^{2}+\gamma^{2}\left(s_{z}-v t\right)^{2}}}\right|_{\mathbf{s}=0} .
\end{aligned}
$$


So far we have dealt with the full Taylor expansion. The truncation introduced by Bertulani et al [5] was to keep only the terms in Equation (6) in which $\ell=\lambda$. This implies the approximation

$$
\Phi(\mathbf{r}, t) \sim \sum_{\lambda, \mu} B_{\mu}^{\lambda, \lambda} r^{\lambda} Y_{\mu}^{\lambda}(\hat{r}) \quad \text { TTEA. }
$$

Note that in the non-relativistic limit, in which $\gamma \sim 1$, we have

$$
\nabla_{\mathbf{s}}^{2} \frac{1}{\sqrt{\left(s_{x}-b_{x}\right)^{2}+\left(s_{y}-b_{y}\right)^{2}+\left(s_{z}-v t\right)^{2}}}=0
$$

if $\mathbf{s}=0$ and $b \neq 0$. In this case, all the $B_{\mu}^{\lambda, \ell}$ vanish except those with $\ell=\lambda$. But if $\gamma>1$

$$
\nabla_{\mathbf{s}}^{2} \frac{1}{\sqrt{\left(s_{x}-b_{x}\right)^{2}+\left(s_{y}-b_{y}\right)^{2}+\gamma^{2}\left(s_{z}-v t\right)^{2}}} \neq 0
$$

and we no longer have the $\ell=\lambda$ selection rule. Thus the $\ell=\lambda$ truncation introduced by Bertulani et al is a significant extra assumption.

\section{Equivalence of the LWLA and TTEA}

We have seen that LWLA and TTEA both make exactly the same truncation $(\ell=\lambda)$ in the expansion of $\Phi(\mathbf{r}, t)$ in terms of $r^{\ell} Y_{\mu}^{\lambda}(\hat{r})$. Therefore the two approximations are equivalent.

Since the vector potential associated with $\Phi(\mathbf{r}, t)$ is $\mathbf{A}(\mathbf{r}, t)=\frac{v}{c} \Phi(\mathbf{r}, t) \hat{z}$, the equivalence of LWLA and TTEA for the scalar potential implies that these approximations are also equivalent when applied to the vector potential.

The merit of the TTEA is that it directly yields explicit expressions for $\Phi(\mathbf{r}, t)$ and $\mathbf{A}(\mathbf{r}, t)$. However, it is important to keep in mind that results calculated with these expressions have exactly the same range of validity as results calculated with the LWLA.

\section{ACKNOWLEDGEMENTS}

One of us (B.F.B.) acknowledges financial support from INFN

\section{References}

[1] A. Winther and K. Alder, Nucl. Phys. A 319 (1979) 518.

[2] C.A. Bertulani and G. Baur, Phys. Rep. 163299 (1988).

[3] H. Emling, Progr. Part. Nucl. Phys. 33792 (1994).

[4] Ph. Chomaz and N. Frascaria, Phys. Rep. 252, 275 (1995). 
[5] C.A. Bertulani, F. Canto, M.S. Hussein and A.F.R. de Toledo Piza, Phys. Rev. C 53 (1996) 334

[6] E.G. Lanza, M.V. Andrés, F. Catara, Ph. Chomaz and C. Volpe, Nucl. Phys. A 613 (1997) 445.

[7] T. Aumann, P.F. Bortignon and H. Emling, Annu. Rev. Nucl. Part. Sci. 48 (1998)

[8] J.D. Jackson, Classical Electrodynamics (Wiley, New York, 1975).

[9] B.F. Bayman and F. Zardi, Phys. Rev. C 59 (1999) 2189. 